\title{
Role of gastrointestinal factors in hunger and satiety in man
}

\author{
B Y N. W. READ \\ Centre for Human Nutrition, University of Sheffield, Northern General Hospital, Herries Road, \\ Sheffield $557 \mathrm{AU}$
}

It is possible that the amount of food consumed by a free-living person in an affluent society is influenced more by social conditioning and emotional factors than by physiological control. This makes it difficult to investigate the mechanisms of food intake in humans; there are so many possible factors to consider.

Many would consider that it is easier to study eating behaviour in experimental animals. At least the social and psychological factors can be controlled by keeping the animals in solitary confinement and feeding a monotonous diet. But surely rats have feelings too. They may not like being confined; rats are sociable animals and may suffer from a solitary existence, and they may not particularly relish rat cake every day. It may be misleading to regard such observations as relevant to humans in a free-living state. And there are other problems; species differences, for example. To what extent can animals provide insights into the mechanisms of eating behaviour in humans? Do people eat like pigs or like dogs or like rats? I suppose it depends on the people. But perhaps the major limitation to animal experiments is that animals cannot report how hungry or how full they feel. This is important because people (and perhaps other animals) eat for reasons other than the physiological need for food.

Eating behaviour is complicated and it is important to consider the psychophysiological forces that control it. These are hunger, appetite, satiety and aversion. Hunger is the word that is usually applied to the sensation of needing food. Appetite is a more hedonic term; it encompasses the enjoyment of eating, the desire to eat and may be applied to the desire to eat certain food items. Satiety is the feeling of satisfaction that is usually associated with the cessation of food intake. Aversion is the intense dislike of food or certain food items. Hunger and satiety are arguably more to do with physiological control. Appetite and aversion are heavily influenced by psychological factors.

Some would say that the hypothalamic and gastrointestinal mechanisms controlling eating behaviour are buried under so many layers of cognitive and conditioning factors that they are barely discernible. Our position is different; we would say that eating behaviour is under physiological control in much the same way as vomiting or defecation, but like those functions, it can be heavily modulated by psychological factors. It is important for the clinician not to underestimate the influence of the physiological control of hunger and satiety because disruption of those mechanisms may be responsible for the severe disturbances in energy and nutrient balance seen in some patients with metabolic, neurological and gastrointestinal disease.

\section{CONTROL FROM THE GASTROINTESTINAL TRACT}

Humans are batch feeders; they tend to eat in discrete meals separated by periods of 3-4 $h$ in which little or no food is eaten. Although this type of eating behaviour is influenced 
by social factors, the periodicity is compatible with the design of the gastrointestinal tract and corresponds to the time that it takes to process a meal. The physiological function of the stomach as a container or hopper is ideally suited for periodic feeding. After ingestion of a meal, the stomach stores and homogenizes the meal and then delivers the mixture at a steady rate into the small intestine for chemical digestion and absorption. At the end of $4 \mathrm{~h}$, most if not all of a standard meal has left the stomach and the majority of this has been absorbed (Malagelada, 1981). At this time, the upper gastrointestinal tract is ready to accept another delivery of food.

Two control mechanisms are necessary to regulate the input of more food into the processor; one to stop ingestion of food when the processor is full and the other to initiate eating when it is empty.

What causes people to stop eating? When food is present in abundance, the factor that most commonly causes most people of normal weight to stop eating is a feeling of fullness or satiation. Most of us eat until we feel replete; we eat to comfort. The ancient Romans may have feasted to a level of discomfort which they could only relieve by vomiting but nowadays this would be regarded as a sign of a severe eating disorder. Obese people and bulimics often continue eating up to the level of extreme discomfort.

It seems almost self-evident that fullness or satiety is signalled by gastric distension. Studies in experimental animals have shown that the stomach is well endowed with stretch receptors which increase their rate of discharge as the organ is distended (Paintal, 1954). Food consumption in obese people can be considerably reduced by inflating a plastic balloon the shape of a small rugby ball in the stomach (Nieben \& Harboe, 1982). Nevertheless the sensation induced by distending a balloon in the stomach is not the same as that experienced at the end of the meal (M. Khan, unpublished results). The latter is often a comfortable feeling of satisfaction whereas gastric distension induces an uncomfortable feeling of gastric pressure.

It is possible that the true sensation of satiety is induced by a combination of input from gastric tension receptors and intestinal chemoreceptors. Consumption of guar gum by itself does not induce feelings of satiety but will do if given in combination with food containing readily available micronutrients (S. French, unpublished results). Inflation of a gastric balloon will only induce a feeling of satiety if this is combined with infusion of lipid into the duodenum (M. Khan, unpublished results). Finally, inspection of our records shows that when gastric emptying is delayed, satiety does not occur upon completion of a meal but this feeling increases rapidly as the gastric contents enter the small intestine (Sepple \& Read, 1989).

Infusion of lipid into the small intestine of human volunteers during consumption of a meal induces a premature feeling of satiety and a reduction in food intake (Welch et al. 1985). Since the presence of lipid in the small intestine delays gastric emptying (Read \& Houghton, 1989), this observation could, at first sight, be explained by suppression of gastric emptying and greater gastric distension during the period of ingestion were it not for the fact that very little solid food normally empties from the stomach during ingestion.

It has been suggested that the release of cholecystokinin (CCK) from the nutrient stimulation of the duodenum may sensitize gastric tension receptors (Lorez \& Goldman, 1982). Another possibility is that the fundic relaxation, caused by the presence of lipid in the small intestine, could alter the sensitive stretch receptors by altering the shape of the stomach. Recent observations have shown that the stomach is less sensitive to distension 
after lipid infusion into the duodenum (M. Khan, unpublished results). Moreover, gastric distension does not cause satiety by itself, even at the highest levels. We suspect an integration of chemoreceptor and mechanoreceptor input at the level of the brain stem.

What causes people to start eating again? This is a more difficult question. Hunger is often regarded as the reciprocal of satiety. In many studies, hunger and satiety are scored on a continuous scale with 'as hungry as I could possibly be' at one end and 'as full as I could possibly be' at the other. Yet, when the two sensations are scored independently, fullness can decline to zero before hunger begins to increase.

There is no proof that the two different sensations are determined by the same mechanisms. The presumption that the sensation of hunger is unmasked by a reduction in input from gastric tension receptors as the stomach empties seems very unlikely. Eating behaviour does not operate to maintain a near constant level of gastric fullness. Only a very small proportion of the subjects in our studies reported hunger shortly after the stomach had started to empty; most did not feel like anything to eat until the stomach was nearly empty (Sepple \& Read, 1989). Eating a meal usually abolishes hunger, but if the meal remains in the stomach, then hunger is often not abolished even though it may become uncomfortable to eat any more (Sepple \& Read, 1989). Distending a balloon in the stomach fails to relieve hunger (M. Khan, unpublished results). These observations strongly suggest that gastric distension is not an important factor in signalling the sensation of hunger.

More recent observations suggest that the onset of hunger may be mediated or at least heavily modulated by chemical stimulation of the small intestine. Infusion of lipid solutions into the duodenum reduced the sensation of hunger before a meal and slowed the rate of ingestion (Welch et al. 1988). This result contrasted with the effect of the same infusion lower down in the ileum (Welch et al. 1985), which only induced early satiety and did not affect preprandial hunger and eating rate, supporting the notion that hunger and satiety are influenced by separate control mechanisms. When the same lipid solutions were infused into the bloodstream (Welch et al. 1988), hunger and satiety were not affected, suggesting that lipid in the small intestine modulates eating behaviour by an action in intestinal lipid receptors (Welch et al. 1988). The suppression of hunger by duodenal lipid may explain why eating a bar of chocolate may suppress hunger (spoil the appetite) but only after a delay of about 15-30 min which may correspond to gastric emptying. The effect of the chocolate is transient, probably because it takes only a short time for the nutrients to be absorbed into the small intestine.

These conclusions are supported by the more physiological experiment showing that enrichment of a breakfast with fat without any change in amount of food consumed suppresses feelings of hunger for lunch $4 \mathrm{~h}$ later and reduces food consumption at that meal (Sepple \& Read, 1990). The amount of food remaining in the stomach immediately before lunch was no greater after consumption of the high-fat breakfast compared with the low-fat breakfast. The major difference was that plasma triacylglycerol levels were still markedly elevated at lunchtime after the high-fat breakfast, indicating continued absorption of lipid and, hence, continued stimulation of lipid receptors in the small intestine or perhaps in the liver.

The presence of nutrients in the small intestine suppresses the periodic pattern of contractile activity known as the migrating motor complex, replacing it with an irregular pattern of contractions that remains until the nutrient content of the small intestine 
declines (Weisbrodt, 1986). The temporal association between the increase in hunger scores and the return of the small intestinal migrating motor complex observed in our volunteer subjects (Sepple \& Read, 1989) is further evidence in favour of mediation by intestinal nutrient receptors.

If, as the evidence suggests, the interaction of nutrients with receptors in the small intestine plays a major role in the control of eating behaviour in humans, then this is likely to do so through the release of chemical transmitters from chemoreceptive or taste cells in the small intestinal epithelium. In recent years attention has focused on CCK. This peptide is stored in the epithelium of the duodenum and released by lumen lipid and peptides. It suppresses eating behaviour when infused into the bloodstream of normal volunteers (Kissileff et al. 1981). Administration of specific CCK antagonists increases food consumption in human volunteers (Wolkowitz et al. 1990). All these findings strongly suggest that CCK does indeed mediate hunger and satiety in humans, but what is the site of action? Does CCK act through the bloodstream as a hormone, does it act locally as a paracrine substance or does it act directly on the brain as a neurotransmitter? In order to answer this question, we compared post-prandial plasma levels of CCK with sensations of hunger and fullness in eight healthy volunteers (S. J. French, B. Murray, R. D. E. Rumsey, C. P. Sepple and N. W. Read, unpublished results). Our results showed that while the average profiles would support a possible role for CCK as a hormone, with high CCK shortly after a meal when the subjects felt full falling to low levels when the subjects felt hungry, inspection of the profiles from individual subjects showed considerable variation. In some subjects the plasma CCK was very low immediately after a meal and rose when subjects started to feel hungry. The only consistent finding was that the release of CCK into the blood was strongly correlated with gastric emptying, being high when gastric emptying was rapid and flat when gastric emptying was slow, compatible with the limitation of CCK release by delivery from the stomach. Although the results did not support a role for CCK as a satiety or hunger hormone, they did not rule out a role for CCK as a paracrine agent or neurotransmitter.

\section{SUMMARY}

There is, we feel, a strong case to be made for a major influence of gastrointestinal factors in the control of eating behaviour in humans. Moreover, the findings suggest that satiety and hunger are controlled by different gastrointestinal mechanisms. Gastric distension is perhaps the most important factor that stops people eating, but a true feeling of satiety also requires the presence of nutrients in the small intestine. Hunger appears to be little influenced by the amount of food in the stomach and appears to be more dependent on the nutrient load in the small intestine.

I do not mean by this to suggest that the gastrointestinal mechanisms are the only mechanisms controlling eating behaviour in humans or even the most important. Nevertheless they constitute an important and fundamental control system that links with the post-absorptive mechanisms and a plethora of social and conditioning factors to influence what we eat.

\section{REFERENCES}

Kissileff, H. R., Pi-Sunyer, F. X., Thornton, J. \& Smith, G. P. (1981). C-terminal octapeptide of cholecystokinin decreases food intake in man. American Journal of Clinical Nutrition 34, 154-160. 
Lorez, D. \& Goldman, S. (1982). Vagal mediation of cholecystokinin effect in rats. Physiology and Behavior 29, 599-604.

Malagelada, J.-R. (1981). Gastric, pancreatic and biliary responses to a meal. In Physiology of the Gastrointestinal Tract [L. R. Johnson, editor]. New York: Raven Press.

Nieben, O. G. \& Harboe, H. (1982). Intragastric balloon as an artificial bezoar for treatment of obesity. Lancet i, 198-199.

Paintal, A. S. (1954). A study of gastric stretch receptors. Their role in the peripheral mechanism of satiation of hunger and thirst. Journal of Physiology 126, 255-270.

Read, N. W. \& Houghton, L. A. (1989). Gastric emptying. Gastroenterology Clinics of North America 18, 359.

Sepple, C. P. \& Read, N. W. (1989). Gastrointestinal correlates of the development of hunger in man. Appetite 13, 183-191.

Sepple, C. P. \& Read, N. W. (1990). The effect of pre-feeding lipid on food intake and satiety in man. Gut 31, $158-161$.

Weisbrodt, N. W. (1986). Motility of the small intestine. In Physiology of the Gastrointestinal Tract [L. R. Johnson, editor]. New York: Raven Press.

Welch, I. McL., Saunders, K. \& Read, N. W. (1985). The effect of ileal and intravenous infusions of fat emulsions on feeding and satiety on human volunteers. Gastroenterology 89, 1293-1297.

Welch, I. McL., Sepple, C. \& Read, N. W. (1988). Comparisons of the effect of infusion of lipid into the jejunum on eating behaviour and satiety in man. Gut 29, 306-311.

Wolkowitz, O. M., Gertz, B., Weingartner, H., Beccaria, L., Thompson, K. \& Liddle, R. A. (1990). Hunger in humans induced by MK-329, a specific peripheral-type cholecystokinin receptor antagonist. Biological Psychiatry 28, 169-173. 